\title{
Spectrophotometry of Selected AGN Seyfert Galaxy AKN 564
}

\author{
L.S. Slavcheva, B.M. Mihov, G.T. Petrov, R.S. Bachev \\ Institute for Astronomy, Bulgarian Academy of Sciences, \\ lslav@astro.bas.bg
}

Akn $564\left(\alpha_{1950}=22^{h} 40^{m} 18.3^{s}, \delta_{1950}=29^{\circ} 27^{\prime} 47^{\prime \prime}\right)$ is a Sy1.5G SBb type galaxy. According to Zwicky (1966) it has a photographic magnitude $\mathrm{m}_{\mathrm{p}}=14.4$ and a redshift of 0.025 . The spectra of the galaxy were obtained at the $2.6-\mathrm{m}$ telescope of the Crimean Astrophysical Observatory with a spectrograph having a dispersion of $100 \mathrm{~A} \mathrm{~mm}^{-1}$. They were processed with the help of SPEC and LONG packages integrated in MIDAS. As a result of the spectrophotometry we obtain the fluxes at $\lambda \lambda 4363,4959,5007 \mathrm{~A}: \mathrm{I}(4363), \mathrm{I}(4959), \mathrm{I}(5007)$. The spectrum of the galaxy in $\lambda \lambda 4000-7000$ is shown in Figure 1 . We use the relation of the fluxes of those narrow forbidden emission lines:

$$
\begin{gathered}
R=[j(\lambda 4959)+j(\lambda 5007)] / j(\lambda 4363) \\
=\left[8.32 \exp \left(3.29 \times 10^{4} / T\right] /\left(1+4.5 \times 10^{-4} N e / T^{1 / 2},\right.\right.
\end{gathered}
$$

sensitive at a greater extent to the electron temperature $T_{e}$ than to the electron density $n_{e}$. The value of $R=74.3$ we got, having a typical value of $n_{e}$ $=5 \times 10^{5} \mathrm{~cm}^{-3}$ for the NLR (Narrow Line Region), leads to the estimation of a typical temperature of $\mathrm{T}_{e}=10^{4} \mathrm{~K}$.

We can evaluate the effective volume $V_{\text {eff }}$ and respectively the size $R_{\text {eff }}$, the mass $M_{g}$ and the kinetic energy $E_{k}$ of the emitting gas in the NLR with $n_{e}=$ $5 \times 10^{5} \mathrm{~cm}^{-3}$ and $\mathrm{T}_{\mathrm{e}}=10^{4} \mathrm{~K}$ assumed and $\mathrm{I}(5007)$ measured via the equations (Dibay 1980):

$$
\begin{gathered}
L\left(H_{\beta}\right)=4 \pi R^{2}(1+z)^{2} I\left(H_{\beta}\right) ; \\
V_{\mathrm{eff}}=R^{2} I\left(H_{\beta}\right) / j\left(H_{\beta}\right) ; \\
R=c z / H ; \\
V_{\mathrm{eff}}=f V ; \\
R_{\mathrm{eff}}=\left(3 V_{\mathrm{eff}} / 4\right)^{1 / 3} ; \\
M_{\mathrm{g}}=n_{\mathrm{e}} m_{\mathrm{p}} V_{\mathrm{eff}} / M_{\mathrm{o}} ; \\
E_{\mathrm{k}}=1 / 2 M_{\mathrm{g}} v^{2}=1 / 4 M_{\mathrm{g}} F W H M ; \\
M_{\mathrm{c}}=3 v_{\mathrm{v}}^{2} R / G,
\end{gathered}
$$

where $\mathrm{V}$ is the geometrical volume of the region, $\mathrm{f} \approx 10^{-3}$ is the filling factor and $\mathrm{j}$ is the emmission coefficient.

$T_{e}$ and $n_{e}$ in the BRL (Broad Line Region) cannot be estimated directly. We accept representative of the BLR values of $\mathrm{n}_{e}=5 \times 10^{5} \mathrm{~cm}^{-3}$ and $\mathrm{T}_{\mathrm{e}}=$ 
$10^{4} \mathrm{~K}$ acquired by comparing photoionizational models with some observational parameters. As a result we evaluate $V_{\text {eff }}, R_{e f f}, M_{g}, E_{k}$ and the mass of the central object $M_{c}$, all of them given in the following table:

\begin{tabular}{|c|c|c|c|}
\hline \multicolumn{2}{|l|}{ NLR } & \multicolumn{2}{|l|}{ BLR } \\
\hline $\mathrm{n}_{\mathrm{e}},\left[\mathrm{cm}^{-3}\right]$ & $5 \times 10^{5}$ & $\mathrm{n}_{\mathrm{e}},\left[\mathrm{cm}^{3}\right]$ & $10^{9}$ \\
\hline$T_{e},[K]$ & $10^{4}$ & $\mathbf{T}_{e},[\mathrm{~K}]$ & $10^{4}$ \\
\hline $\mathrm{I}([\mathrm{OIm}] \lambda 5007),\left[\mathrm{erg} \mathrm{cm}^{-2} \cdot \mathrm{s}^{-1}\right]$ & $1.04 \times 10^{-12}$ & $\mathbf{I}\left(\mathbf{H}_{\beta}\right),\left[\mathrm{erg}_{\mathrm{c}} \mathrm{cm}^{-2} \cdot \mathrm{s}^{-1}\right]$ & $5.85 \times 10^{-13}$ \\
\hline FWHM $([0 \mathrm{OII}] \lambda 5007),\left[\mathrm{cm} \cdot \mathrm{s}^{-1}\right.$ & $663 \times 10^{5}$ & FWHM $\left(\mathrm{H}_{\beta}\right),\left[\mathrm{cm} . \mathrm{s}^{-1}\right]$ & $899 \times 10^{5}$ \\
\hline $\mathrm{L}([\mathrm{OW}] \lambda 5007),\left[\mathrm{erg} . \mathrm{s}^{-1}\right]$ & $9.18 \times 10^{41}$ & $\mathrm{~L}\left(\mathbf{H}_{\beta}\right),\left[\right.$ erg.s. $\left.{ }^{-1}\right]$ & $5.18 \times 10^{41}$ \\
\hline $\mathrm{j}([\mathrm{OW}] \lambda 5007),\left[\mathrm{erg} . \mathrm{cm}^{-3} \cdot \mathrm{s}^{-1}\right]$ & $1.15 \times 10^{-19}$ & $\mathrm{j}\left(\mathbf{H}_{\beta}\right)$, [erg.cm $\left.\mathrm{cm}^{-3} \mathrm{~s}^{-1}\right]$ & $6.63 \times 10^{-9}$ \\
\hline$V_{\mathrm{e} \pi},\left[\mathrm{cm}^{3}\right]$ & $1.6 \times 10^{55}$ & $\mathrm{~V}_{\mathrm{en},},\left[\mathrm{cm}^{3}\right]$ & $6.19 \times 10^{48}$ \\
\hline $\mathrm{R},[\mathrm{pc}]$ & 5 & $\mathbf{R},[\mathrm{pc}]$ & 0.037 \\
\hline $\mathbf{M}_{\mathbf{0}},[\mathrm{Mo}]$ & $6.68 \times 10^{3}$ & $\mathbf{M}_{\mathrm{g},}[\mathrm{Mo}]$ & 5.17 \\
\hline$E_{k},[e r g]$ & $7.34 \times 10^{51}$ & $E_{k},[$ erg] & $1.04 \times 10^{49}$ \\
\hline & & $\mathbf{M}_{c}[\mathrm{Mo}]$ & $0.52 \times 10^{7}$ \\
\hline
\end{tabular}

The errors of the fluxes are about $7 \times 10^{-15} \mathrm{erg} \mathrm{cm}^{-2} \mathrm{~s}^{-1}$ and the errors of the other parameters are about $10-30 \%$. 


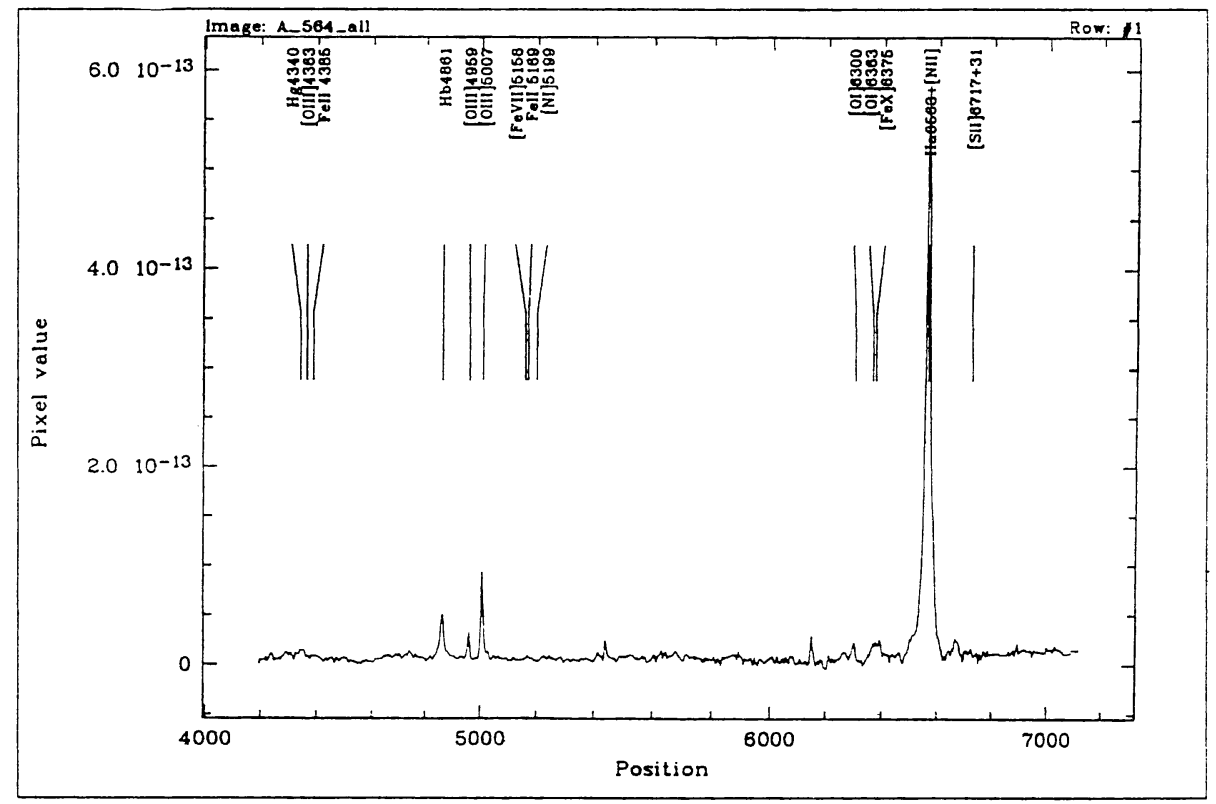

Figure 1. Energy distribution in $\lambda \lambda$ 4000-7000 A for Akn564. The data reduction was made by MIDAS $95 \mathrm{NOV}$ packages. The strongest forbidden and permitted lines are marked.

\section{References}

Arakelian, M., 1975, Publ.Bjurak.Obs., 47, 3

Dibay, E., 1980, Astron. J., , 57, 677

Zwicky, F., 1966, Ap.J., , 143, 192 